\title{
La pronunciación en la enseñanza de lenguas extranjeras
}

\author{
Tania Silverio-Pérez*
}

\begin{abstract}
Resumen
El tratamiento de la dimensión fonética del lenguaje ha estado marginado en las clases de español como lengua extranjera (ELE). Aunque hubo un intento de reivindicarlo, no se ha logrado materializar eficientemente en el contexto docente. Factores como la desestimación de la pronunciación cuando se enseña la lengua en su medio, la complejidad del contenido que le compete y la desorientación metodológica de los profesores para trabajarla condicionan esta realidad. Por tanto, es necesario partir de la revisión del concepto dentro de la didáctica de las lenguas extranjeras ( $\mathrm{LE}$ ) como fenómeno en sí y como contenido que debe enseñarse y corregirse. Este trabajo tiene como objetivo reflexionar sobre los conceptos pronunciación, enseñanza de la pronunciación y corrección fonética a partir de teorías precedentes. Para esto, se tuvo en cuenta la obra de Antich et al., un clásico en la metodología de la enseñanza de LE en Cuba, y de otros investigadores que han desarrollado el tema desde el español, fundamentalmente. Se asume que la pronunciación es una habilidad que ha de enseñarse y desarrollarse en el proceso de enseñanza-aprendizaje de la LE para que el estudiante, como parte de su competencia comunicativa, alcance una competencia fónica.
\end{abstract}

Palabras clave: corrección, enseñanza, lenguas extranjeras, pronunciación.

\section{Pronunciation in Foreign Language Teaching}

\begin{abstract}
Treatment of the phonetic aspect of language has been marginalized in classes of Spanish as a Foreign Language (sFL). Although there has been an attempt to reestablish its importance, this has not lead to effective, material changes in teaching. Factors such as undervaluing pronunciation in settings of language teaching, the complexity of the corresponding content, and the methodological disorientation of teachers have all contributed to the current situation. We must therefore revisit the concept in foreign language teaching as a phenomenon within itself, and as content that should be taught and corrected. This study seeks to reflect on the concepts of pronunciation, pronunciation teaching, and phonetic correction based on earlier theories, including the work of Antich et al. - a classic text in the area of foreign language teaching methodology in Cuba - and other researchers in this area, primarily in relation to Spanish. It is assumed that pronunciation is an ability that must be taught and developed in the foreign language teaching-learning process in order for students to achieve phonetic competence as part of their communicative competence.
\end{abstract}

Keywords: correction, teaching, foreign languages, pronunciation.

\section{A pronúncia no ensino de línguas estrangeiras}

* Magíster en Lingüística Hispánica. Profesora del Departamento de Español, Universidad de La Habana, La Habana, Cuba.

Correo electrónico:

tanias@rect.uh.cu

Recibido: 18 de julio del 2014

Aprobado: 28 de octubre del 2014

Cómo citar este artículo: Silveiro-Pérez,

Tania "La pronunciación en la enseñanza de lenguas extranjeras". Rastros Rostros 16.30 (2014): 57-62. Impreso. doi: http://dx.doi. org/10.16925/ra.v16i30.818

\section{Resumo}

O tratamento da dimensão fonética da linguagem esteve marginalizado nas aulas de espanhol como língua estrangeira (ELE). Mesmo havendo uma tentativa de reivindicá-lo, não se conseguiu materializar eficientemente no contexto docente. Fatores como a denegação da pronúncia quando se ensina a língua em seu meio, a complexidade do conteúdo que compete a ela e a desorientação metodológica dos professores para trabalhá-la condicionam essa realidade. Portanto, é necessário partir da revisão do conceito dentro da didática das línguas estrangeiras (LE) como fenômeno em si e como conteúdo que deve ser ensinado e corrigido. Este trabalho tem como objetivo refletir sobre os conceitos pronúncia, ensino da pronúncia e correção fonética a partir de teorias precedentes. Para isto, levou-se em conta a obra de Antich et al., um clássico na metodologia do ensino de LE em Cuba, e de outros pesquisadores que desenvolveram o tema para o ensino de espanhol, fundamentalmente. Assume-se que a pronúncia é uma habilidade que deve ser ensinada e desenvolvida no processo de ensino-aprendizagem da LE para que o estudante, como parte de sua competência comunicativa, alcance uma competência fônica.

Palavras-chave: correção, ensino, línguas estrangeiras, pronúncia. 


\section{Introducción}

La comunicación es esencialmente oral. En La enseñanza de la lengua materna en la escuela primaria se plantea:

La lengua genuina, la verdadera lengua, es la que se oye y se habla; y lo es como hecho primero del lenguaje; y lo es como instrumento básico de expresión y comunicación ...; en consecuencia parece que es el instrumento que habría de tender primordialmente a desarrollar y vigorizar. En sí la lengua escrita es una soberana conquista del hombre ... ; en el proceso de la enseñanza del idioma hay que tener en cuenta que la lengua escrita es un arte derivado, el cual cumplirá mejor su oficio suplente cuanto más nutrido esté de rica y vigorosa lengua hablada, que es su básico modelo. (44)

Sobre el mismo tema, pero referido a la enseñanza de la lengua extranjera (LE), Antich, Gandarias y López reconocen la primacía del lenguaje oral que se basa "en la realidad objetiva de su naturaleza como fenómeno social y medio de comunicación verbal por excelencia" (62). Por tanto, la prioridad se da al establecimiento de mecanismos de comprensión y producción oral. La escritura, salvo en cursos de especialización en el idioma, tiene un carácter instrumental o auxiliar, más que un fin en sí misma.

Según Cantero, "es un error considerar lo oral y lo escrito como las dos caras de una misma moneda: ... deben considerarse dos monedas distintas, separadas y distintas que ni siquiera tienen el mismo valor" (Didáctica de la pronunciación 6). El autor también reconoce como lengua natural y primera, la oral.

Para la mayoría de los profesores de LE, es evidente la importancia de la lengua oral, sin embargo, desestiman la pronunciación, que es su soporte (sin esta no hay lengua oral), la dimensión fónica de la lengua (de la comunicación), entendida como todo el sistema de la forma sonora (sonido, acento, ritmo, entonación; función y significado). Su dominio implica codificarla y descifrarla adecuadamente, de lo contrario no hay comunicación (Cantero "La cuestión del acento").

Cuando se estudia una LE en su medio lingüístico y los profesores la tienen como LM, sucede con frecuencia que el profesor descuida la atención a la pronunciación y la ve como una habilidad que adquirirá el estudiante en su inmersión en el contexto. Otros elementos que favorecen esta situación son la complejidad del contenido que le compete y la desorientación meto- dológica de los profesores para trabajarla en el proceso de enseñanza-aprendizaje. La metodología conductista que ha regido su tratamiento resulta incongruente con los nuevos enfoques metodológicos.

Sin embargo, es importante señalar que si no se enseña conscientemente en la etapa inicial ni se trabaja en la corrección de errores y malos hábitos (de pronunciación) en niveles posteriores el error se fosiliza y difícilmente puede ser eliminado. Lo peor es su incidencia negativa en el desarrollo de la competencia comunicativa del estudiante.

Ante esta situación, es necesario partir de la revisión del concepto pronunciación dentro de la didáctica de las lenguas extranjeras como fenómeno en sí y como contenido que debe enseñarse y corregirse. De esta forma, se favorece la actuación didáctica del profesor en el aula. Este trabajo tiene como objetivo sistematizar los conceptos pronunciación, enseñanza de la pronunciación y corrección fonética a partir de teorías precedentes.

\section{La pronunciación: ¿hábito, destreza o habilidad?}

Ante la existencia de diferentes criterios sobre la definición de hábito, destreza y habilidad, y la importancia de estos conceptos para este trabajo, es necesario aclarar que se asume que:

- El concepto destreza no ha sido suficientemente sistematizado. Aun es utilizado como sinónimo de habilidad y de hábito.

- Existen algunos profesores e investigadores que se oponen alaidentificación de destrezayhábito.Leatribuyen a la primera una naturaleza operacional que no reconocen en la segunda, pero es evidente que el uso de un término u otro responde a la afinidad por una escuela u otra sobre la naturaleza del aprendizaje.

- La publicación del Marco de referencia europeo para el aprendizaje, la enseñanza y la evaluación de lenguas (MCERL) del Instituto Cervantes marca una pauta en la enseñanza de LE y le impone a los tiempos modernos el empleo del término destreza; mas no debe haber un prejuicio que minimice o desdeñe hábito.

- La habilidad es un proceso complejo de acciones y operaciones dominado por el sujeto, que responde a un objetivo. Tiene como base el conocimiento y 
forma parte del proceso de enseñanza-aprendizaje; se perfecciona mediante la sistematización. La habilidad implica un saber hacer.

- El hábito (destreza) está compuesto por acciones y operaciones semi- o completamente automatizadas que, con determinada lógica y consecutividad, dan forma de proceso continuo a la actuación del sujeto. Hábito y habilidad están indisolublemente relacionados y, como procesos, se desarrollan simultáneamente.

Luego de esta aclaración se hará referencia a lo expuesto por algunos autores sobre el tema de la pronunciación. Se respeta la terminología de cada uno.

Antich, Gandarias y López ${ }^{1}$ consideran que la pronunciación es una habilidad que se manifiesta en el proceso real de comunicación e implica "el desarrollo de habilidades en dos aspectos fundamentales: el de audición ... y el de producción del sistema fonológico de la lengua" (235). Cuando ejemplifican los tipos de hábitos, se refieren la producción y percepción de sonidos aislados.

Por su parte, el MCER expone en el subepígrafe 5.1.4.2 las destrezas correspondientes a la pronunciación:

- La capacidad de distinguir y de producir sonidos corrientes y estructuras prosódicas.

- La capacidad de percibir y de concatenar secuencias desconocidas de sonidos.

- La capacidad, como oyente, de convertir (es decir, dividir en partes distintivas y significativas) una corriente continua de sonido en una cadena significativa y estructurada de elementos fonológicos.

- La comprensión o dominio de los procesos de percepción y producción de sonido aplicables al aprendizaje de una nueva lengua.

El MCER aclara que estas destrezas fonéticas generales (que son capacidades, según se plantea) se distinguen de la capacidad de pronunciar una lengua concreta (Instituto Cervantes 102). Aunque establece cierta diferencia entre las partes (destrezas fonéticas) y el todo (habilidad pronunciar), el empleo de la palabra capacidad para ambos conceptos impone cierta

Se parte de este texto porque, aunque fue publicado hace más de 25 años y algunos de sus presupuestos teóricos no están vigentes, ha sido la guía básica para la formación de profesores de LE en Cuba y constituye hasta hoy un clásico en la materia. ambivalencia que impide determinar si habla de hábitos o (micro) habilidades. De cualquier forma, tener la capacidad de hacer algo no implica tener la habilidad de hacerlo. Median el grado de esfuerzo requerido y el resultado.

Cuq y Gruca (citados en Spalacci) también relacionan el término pronunciación con la capacidad de entender y producir el sistema fonológico de la lengua, ${ }^{2}$ por lo que consideran esencial ligar íntimamente en el proceso de enseñanza-aprendizaje del sistema fonológico, la audición o percepción con la articulación. De esta forma, la percepción se ve como complemento significativo y no como parte del proceso en sí.

La pronunciación, vista no como simple articulación, como la producción de los sonidos de una lengua con un ritmo, un acento y una entonación determinados, como una actividad derivada de la lengua escrita (leer) carente de función y significado, sino como un proceso complejo que permite al hablante estructurar su discurso, completar su significado e intención y al oyente el proceso de comprensión auditiva, va más allá de la mera suma de procedimientos automatizados.

Francisco Cantero, en "Adquisición de competencias fónicas", propone una nueva perspectiva teórica en la que describe la pronunciación como un fenómeno multidimensional que tiene su ámbito de acción y de estudio en la competencia comunicativa en su conjunto. ${ }^{3} \mathrm{El}$ autor define los elementos que la configuran en cada una de las subcompetencias comunicativas y nombra la dimensión fónica de cada una de estas, competencia fónica.

A partir de las definiciones anteriores, se asume la pronunciación como una habilidad que ha de enseñarse y desarrollarse en el proceso de enseñanza-aprendizaje de la LE para que el estudiante, como parte de su competencia comunicativa, alcance una competencia fónica.

2 En el texto al que se hace referencia (Spalacci) aparecen especificados 'los contenidos' de la dimensión fónica de la lengua: sonido, ritmo y entonación. Además, se presenta la prosodia como un sistema independiente del fonológico.

3 El autor define competencia comunicativa como "el conjnto de saberes, habilidades y recursos que cada individuo pone en juego para relacionarse con los demás, para comunicarse" (3). Incluye dos tipos de competencias: las competencias estratégicas (se corresponden con los saberes, las habilidades y los recursos que permiten la comunicación -competencias lingüística, discursiva, cultural y estratégica-) y las competencias especificas (referidas a las actividades comunicativas, según la formulación del MCER -competencias productiva, perceptiva, mediadora e interactiva-). 


\section{Enseñanza de la fonética, enseñanza de la pronunciación, corrección fonética}

La fonética es la disciplina que estudia los sonidos que intervienen en la comunicación, cómo son (fonética acústica), cómo se producen (fonética articulatoria) y cómo se perciben (fonética perceptiva):

[su enseñanza] consiste en una reflexión explícita sobre el sistema; suele realizarse en el marco de los estudios de Filología ${ }^{4}$ y se persigue con ella que el futuro especialista adquiera un conocimiento formal y detallado de las características articulatorias, acústicas y perceptivas de los elementos segmentales y suprasegmentales de la lengua. (Llisterri 91)

Es evidente que a un estudiante que aprende una LE con fines comunicativos no le interesa, como regla, este conocimiento, sino desarrollar una competencia comunicativa que le permita la actuación eficiente en diferentes contextos. Para lograrlo es necesario que sepa pronunciar la lengua a fin de que su interacción sea fluida e inteligible, porque la pronunciación hace posible la producción y la percepción de la lengua (oral).

Bartolí asegura que enseñar fonética no es lo mismo que enseñar pronunciación y ninguno de los procesos garantiza el otro.

Llisterri refiere que la enseñanza de la pronunciación permite el desarrollo de una de las habilidades que el estudiante debe dominar: la pronunciación.

Antich, Gandarias y López analizan el fenómeno desde la perspectiva del estudiante y sólo aluden a "la adquisición ${ }^{5}$ de una buena pronunciación" (235). No hablan de aprendizaje ni de enseñanza, sino de corrección de errores. Este criterio se retoma en páginas posteriores.

Por su parte, Cantero define didáctica de la pronunciación como un "enfoque metodológico que integra la pronunciación en los parámetros del Enfoque

\footnotetext{
4 Filología se refiere a las carreras de lenguas: Filología hispánica, Filología francesa, Filología inglesa, etc.

5 El término adquisición es muy controversial en el área de la enseñanza de lenguas extranjeras. Como tendencia general, se asocia al aprendizaje fuera del contexto educativo formal, que ocurre de forma natural (aprendizaje implícito). En el caso de adquisición fónica, en esta investigación se asume la definición dada por Cantero ("Conceptos clave en lengua oral"): "Proceso mediante el cual el individuo es capaz de codificar y descodificar eficientemente el discurso oral según las reglas fónicas de la lengua aprendida. No consiste en el mero aprendizaje del repertorio fonético de la lengua, sino en la integración y la comprensión global del discurso a partir de una competencia fónica suficientemente
} desarrollada." (34).
Comunicativo, dentro del tratamiento de la lengua oral ... Su objetivo, entonces, no es ya la mera 'pronunciación correcta', sino la adquisición de la competencia fónica de la lengua meta" ("Conceptos clave" 149). Es un concepto relativamente nuevo que supera la perspectiva prescriptiva de métodos y enfoques anteriores.

El MCER (Instituto Cervantes 111-112) expone en el capítulo 5:

La competencia fonológica ${ }^{6}$ supone el conocimiento y la destreza en la percepción y la producción de:

- Las unidades de sonido (fonemas) de la lengua y su realización en contextos concretos (alófonos).

- Los rasgos fonéticos que distinguen fonemas (rasgos distintivos; por ejemplo: sonoridad, nasalidad, oclusión, labialidad).

- La composición fonética de las palabras (estructura silábica, la secuencia acentual de las palabras, etc.).

- Fonética de las oraciones (prosodia):

- Acento y ritmo de las oraciones

- Entonación

En estudios posteriores de Cantero, como "Fonética y didáctica de la pronunciación", y en correspondencia con su visión compleja de la comunicación humana, considera que la enseñanza de la pronunciación equivale a "la enseñanza de estrategias que permiten formular adecuadamente y entender un discurso oral genuino y espontáneo, en su conjunto" (561). Para él, la competencia estratégica ${ }^{7}$ es el eje de toda la competencia comunicativa del hablante, la que permite la articulación de todas las demás, incluida la fónica. Define como objetivo prioritario de la enseñanza de la pronunciación, entonces, "conseguir una competencia estratégica que permita al alumno construir una nueva pronunciación en la lengua meta, adecuada al contexto y al interlocutor" (Cantero "Adquisición de competencias" 13).

Mientras este autor se centra en el autodesarrollo del estudiante, en cómo puede lograr una competencia fónica eficiente, Iruela lo hace en la capacidad para usarla. Expone que el objetivo final de la didáctica de

\footnotetext{
6 Los casos en que aparece el término competencia fonológica en lugar de competencia fónica están dados por la fidelidad a la bibliografía referida. En este trabajo se asume el segundo.

$7 \quad$ El autor la define como recursos que el hablante pone en juego para relacionar sus conocimientos y habilidades, con el fin de afrontar cada situación comunicativa.
} 
la pronunciación ${ }^{8}$ debe ser capacitar al estudiante para que pueda utilizar la competencia fonológica de la LE de forma eficiente en situaciones reales de comunicación.

En cuanto a corrección fonética, debe partirse de que es un concepto tradicional que se enmarca en las perspectivas prescriptiva y terapéutica de la enseñanza de LE. Su objetivo es que el estudiante pronuncie "correctamente" y el modelo, cómo se pronuncia la escritura. Esto la relaciona directamente con la ortología (ortoepia), nombre dado tradicionalmente a la corrección fonética en la LM.

Cantero, en "Conceptos clave en lengua oral", define corrección fonética (o fonética correctiva) como la "rama de la fonética que ofrece métodos auxiliares a la enseñanza tradicional de la lengua, para corregir y optimizar la pronunciación de los alumnos (tanto en LM, como en L2 O LE), según las normas de pronunciación correcta" (149). La pronunciación correcta depende de la fidelidad a las reglas: "pronunciación de la ortografía" (métodos directo y de transcripción fonética), "técnicas de articulación" (método audiolingual o fonoarticulatorio); correcta discriminación de los sonidos (método estructural o de pares mínimos) y una percepción óptima de las frecuencias del idioma aprendido (método verbo-tonal).

Desde el enfoque comunicativo, pero en sus inicios, Antich, Gandarias y López le atribuyen dos etapas a la corrección fonética dentro del proceso de adquisición de la pronunciación: la de adquisición del sistema fonológico a través del lenguaje controlado y la de utilización y consolidación del sistema fonológico a través de la expresión espontánea. Proponen como procedimientos de corrección: la articulatoria, por oposiciones fonológicas, la verbo-tonal y por el uso de símbolos fonéticos. La presentación de los tipos de ejercicio (lectura oral, canto, poesía y coro hablado) revela la mediación lectoescritora en el proceso y el peso de la pronunciación de la escritura como la correcta. Estas características llevan a Cantero, en "Fonética y didáctica de la pronunciación", a ubicar la corrección fonética en un segundo momento del proceso: cuando los estudiantes ya están en contacto con la lengua escrita.

8 En este trabajo los términos enseñanza de la pronunciación y didáctica de la pronunciación son utilizados como sinónimos. Ambos se refieren al área de la didáctica (enseñanza) de las LE que tiene como objeto de estudio el proceso de enseñanza-aprendizaje de la pronunciación. En algunos casos, como el de Cantero ("Conceptos clave en lengua oral", "Fonética y didáctica de la pronunciación", "Adquisición de competencias fónicas"), se percibe la diferencia entre el concepto más general y abstracto, didáctica, y el más concreto, enseñanza, referido al proceso como tal.
Raúl Alfonso refiere que a "la 'corrección fonética' tradicional la acompañan los conceptos de error como base de la intervención, dependencia de la escritura, carácter segmental de la pronunciación y una aplicación atávica de la metodología didáctica" (4). Es necesario superar el concepto de error como base de la intervención didáctica.

Cantero, en Didáctica de la pronunciación, ofrece una perspectiva que responde a una maduración del enfoque comunicativo, en la que la eficacia fónica sustituye a la corrección fonética y la competencia fónica a la pronunciación correcta. Según él:

Frente a la corrección fonética tradicional (ocupada sólo de los sonidos o las frases aisladas, dependiente de la lengua escrita y cuyo objetivo es la excelencia en la corrección del alumno), la didáctica de la pronunciación propone un tratamiento global de la lengua oral, centrada no en la producción o la discriminación de sonidos, sino en la expresión y la comprensión oral en su conjunto. ("Conceptos clave" 33)

Enseñanza de la pronunciación y corrección fonética se ubican en dos momentos diferentes del proceso, la primera siempre precede a la segunda; aunque, si se asume la enseñanza de la pronunciación como el desarrollo de la competencia fónica, no se debe hablar de corrección, sino de un proceso que debe ir in crescendo en función de una eficacia fónica.

\section{Conclusiones}

El profesor de LE debe reconocer la importancia de la lengua oral como medio de expresión y comunicación y de la pronunciación como su soporte; esa es la premisa fundamental para que se preocupe por incluirla (a la segunda) en el proceso de enseñanza-aprendizaje.

El hecho de que el proceso de enseñanza-aprendizaje de la LE se realice en su medio lingüístico, no exime al profesor de la responsabilidad de desarrollar la competencia fónica del estudiante. Para esto, debe concebir la pronunciación como una habilidad que ha de enseñarse y desarrollarse en ese proceso.

En el contexto de los nuevos enfoques metodológicos, la enseñanza de la fonética queda fuera de la didáctica de las LE con fines comunicativos, pues no es una reflexión explícita sobre el sistema (fonético-fonológico de la lengua) lo que necesita el estudiante, sino competencia fónica para poder expresar sus ideas de forma oral y comprender las de los demás. 
La enseñanza de la pronunciación debe proveer al estudiante de una competencia estratégica que le permita construir una nueva pronunciación en la LE, según el contexto: una competencia fónica para que el hablante pueda estructurar su discurso, completar su significado e intención, y al oyente, comprender lo que escucha.

Mientras, la corrección fonética pretende corregir y optimizar la pronunciación según las normas de "pronunciación correcta”. En este afán se plantea el error como base de la intervención, la dependencia de la escritura y el carácter segmental de la pronunciación.

Enseñanza de la pronunciación y corrección fonética tienen objetivos diferentes. Le corresponde al profesor definir cuándo aplicar cada una, según sus concepciones metodológicas y el contexto en que se inserte el proceso de enseñanza-aprendizaje de la LE. Mas si se asume que la comunicación exige más de eficacia que de corrección, entonces la corrección fonética debe despojarse de su apego a la escritura y responder a la eficacia comunicativa.

\section{Referencias}

Alfonso, Raúl. "Sobre el vocalismo y la pronunciación”. Phonica 1, 2005. Web.

Antich, Rosa, Dariela Gandarias y Emma López. Metodología de la enseñanza de Lenguas extranjeras. La Habana: Editorial Pueblo y Educación, 1987. Impreso.
Bartolí, Marta. "La pronunciación en la clase de lenguas extranjeras". Phonica 1 (2005). Web.

Cantero, Francisco José. "La cuestión del acento en la enseñanza de lenguas". Problemas y métodos en la enseñanza del español como lengua extranjera. Eds. Jesús Sánchez Lobato y Isabel Santos Gargallo. Madrid: S.G.E.L., 1994. Web.

. "Conceptos clave en lengua oral". Conceptos clave en didáctica de la lengua y la literatura. A. Mendoza, coord. Barcelona: Horsori, 1998. Web.

“Fonética y didáctica de la pronunciación”. Didáctica de la lengua y la literatura. A. Mendoza, coord. Madrid: Prentice Hall, 2003. 545-572. Electrónico.

“Adquisición de competencias fónicas”. 2012. Web. Didáctica de la pronunciación. Web.

La enseñanza de la lengua materna en la escuela primaria. Ciudad de La Habana: Editorial Pueblo y Educación, 2001. Impreso.

Iruela, Agustín. "Principios didácticos para la enseñanza de la pronunciación en lengua extranjera”. MarcoELE, Revista de Didáctica (2007). Web.

Instituto Cervantes. Marco de referencia europeo para el aprendizaje, la enseñanza y la evaluación de lenguas. 2002. Web.

Llisterri, Joaquín. "La enseñanza de la pronunciación”. Revista del Instituto Cervantes, 4.1 (2003):91-114.Web.

Spalacci, Mireille. "Enfoque oral y recursos digitales para un curso de francés como lengua extranjera”. Tesis doctoral. Universitat de Barcelona, 2011. Impreso. 